Research

\title{
Sport and sustainable development in Botswana: towards policy coherence
}

\author{
Louis Moustakas $^{1}$ (D) Arda Alan Işık ${ }^{1}$
}

Received: 15 October 2020 / Accepted: 27 October 2020

(C) The Author(s) 2020 OPEN

\begin{abstract}
There are increasing connections made between sport and sustainable development, both internationally and within Botswana's national policies. However, these policy documents are vague and overly broad. There is no focus on where or how sport can be positioned to support sustainable development within Botswana's unique national context. Using policy coherence as a theoretical framework, the goal of this paper is to analyse the current policy as well as propose national policy objectives and instruments to support sport's contribution to sustainable development in Botswana. This paper relies on findings generated through semi-structured qualitative interviews, policy documents and academic literature. We propose that sport should be re-oriented to achieve objectives related to economic development, education, and health. Numerous concrete policy instruments are likewise suggested, including investing in small-scale sport infrastructure, public awareness campaigns and better sport educator training. Ultimately, these instruments enhance the coherence of the overall policy and create synergies by addressing multiple overarching objectives simultaneously.
\end{abstract}

Keywords Sport $\cdot$ Sustainable development $\cdot$ Policy coherence $\cdot$ Botswana $\cdot$ Southern africa

\section{Intoduction}

Both at the national and international levels, there has been growing recognition of the potential for sport to contribute to several Sustainable Development Goals (SDGs). Indeed, there is increasing agreement on the areas where sport can contribute to sustainable development goals at the global level [1]. However, most work related to policy, sport and the SDGs has primarily been focused on international organisations. Though some efforts have begun to address policy coherence in sport and how sport can support national-level sustainable development objectives [2-4], national-level policies are often underdeveloped and under-researched.

This is the case within Botswana. Numerous policy documents, including the National Sport Policy, National Youth Policy, and National Development Plans acknowledge the potential of sport to contribute to a wide range of developmental goals [5-7]. Most notably, sport is included in Botswana's Vision 2036 agenda. Vision 2036 is a broad, cross-cutting agenda that aims to transform Botswana from an upper-middle-income to a high-income country. Within this agenda, four key pillars - sustainable economic development, human and social development, sustainable environment, as well as governance, peace and security—are included [8]. Under each pillar, numerous sub-areas are outlined, and sport is

Electronic supplementary material The online version of this article (10.1007/s43621-020-00005-8) contains supplementary material, which is available to authorized users.

Louis Moustakas, I.moustakas@dshs-koeln.de | ${ }^{1}$ Institute for European Sport Development and Leisure Studies, German Sport University, Am Sportpark Müngersdorf 6, 50933 Cologne, Germany.

Discover Sustainability $\quad$ | (2020) 1:5 $\quad$ https://doi.org/10.1007/s43621-020-00005-8 
included under sustainable economic development. Along with these pillars, Vision 2036 explicitly advocates for the promotion of shared prosperity and achievement of the United Nations' Sustainable Development Goals.

Though this body of policy recognises that sport is intertwined with numerous sustainable development objectives, these policies connect sport to an overly wide array of topics, including gender, education, national identity, employment, youth, health, the environment, transport, tourism and economic development. Little to no detail is provided specifying the policy objectives or the instruments that can support those objectives. This leads to a highly incoherent policy environment that not only inhibits the growth of the sport sector but effectively offsets sport's contribution to the country's sustainable development.

Using policy coherence as a theoretical framework, the goal of this paper is to analyse the current policy and propose national policy objectives and instruments to support sport's contribution to sustainable development in Botswana. We rely on findings generated through semi-structured qualitative interviews, policy documents and academic literature. Based on this, we argue that policy should focus on how sport can contribute to a limited number of cross-cutting sustainable development objectives, and subsequently propose instruments to support these objectives.

The article proceeds in three steps. Firstly, the concept of policy coherence and an analysis of how it manifests itself in relation to sport in Botswana are presented. Then, the specific details of our methodology are outlined. Finally, we propose specific policy objectives and instruments to support sport's contribution to sustainable development in Botswana and illustrate how these instruments can support multiple objectives.

\section{Policy (In)coherence: sport and sustainable development in Botswana}

The concept of policy coherence initially gained prominence in the field of international development in the 1990s and early 2000s, but a formal definition of the term has remained elusive. Early on, proponents of policy coherence aimed to improve development outcomes in the global South by countering problems that arose from divergent or incoherent policies in the North [3]. Now, policy coherence is applied to a range of domains outside of international development. Essentially, policy coherence means that various policies fit together because they share ideas or objectives [9]. In other words, it is about "eliminating conflict and promoting synergy" [10]. Policy coherence can be understood both vertically and horizontally. Vertical coherence refers to common objectives and synergies that exist between policies at different levels of government, be it international, national, regional, or local. Horizontal coherence refers to coordination across policy within a single level of government. For example, the Ministry of Youth, Sport, and Culture and the Ministry of Basic Education might coordinate objectives and instruments related to youth education.

To support the analysis of policy coherence, Nilsson et al. [11] designed a policy-analytical framework, presented in Fig. 1, that distinguishes between policy integration, contextual factors and policy coherence. In this framework, policy coherence is defined by the interaction between policy objectives, instruments, and implementation practices. Here, instruments should

Policy Analytical Framework - Adapted from Nilsson et al. [11]

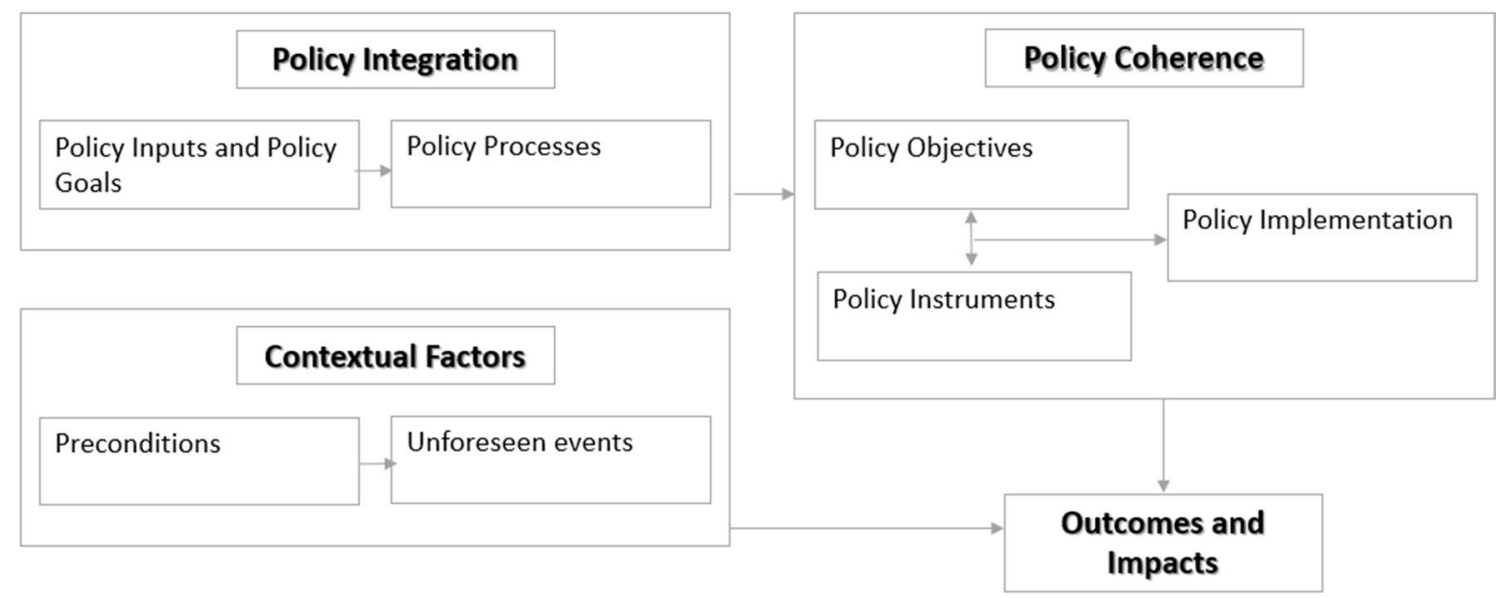

Fig. 1 Policy coherence in a policy-analytical framework, adapted from Nilsson et al. [11] 
be understood as financial, promotional, motivational, regulatory or administrative tools that governments employ to achieve policy objectives [12]. Distinguishing between these three elements allows for the assessment of overall coherence, how policy objectives are conceptualised and how instruments are implemented. Specifically, Nilsson et al. [11] recommend three distinct steps to support the analysis of policy coherence: (1) create an inventory of policy objectives; (2) conduct screening of policy interactions; and (3) do an in-depth mapping of key policy interactions and implementation practices.

Though Botswana, with its Vision 2036 Agenda and National Development Plans, is fairly explicit about the overarching goals it wants to achieve, the country has poorly conceptualised how sport can support specific objectives and what policy instruments should be used. In other words, though sport has been shown to have the potential to support numerous sustainable development goals [1], Botswana policy does not make clear where or how sport should contribute. As discussed above, sport is tied to a large range of sustainable development objectives, and no concrete guidance is provided regarding how sport should be used as an instrument to achieve policy objectives. Following Nilsson [11], we have first created an inventory of policy objectives and instruments related to sport in Botswana, and present this in Table 1. Three facts become evident within this table. First, there is some level of consistency related to the objectives connected to sport. Most policies focus on health, economic development, youth, and national unity. Second, these objectives are nebulously defined. The policy documents do not provide defined or measurable sub-objectives. Finally, the proposed policy instruments-variously described as strategies or policy initiatives-significantly impede policy coherence. The instruments are vague if presented at all, and the distinctions between objectives and instruments are often murky. For instance, in the case of the National Youth Policy, new objectives such as environmental awareness or informal education appear within the proposed strategies.

Policy within the sport sector itself is similarly incoherent. The most recent sport policy in Botswana dates back to 2001. Though as recently as 2014 it was described by the Minister of Sport as "sufficient" [14], this policy does not address the goals of the Vision 2036 agenda or reflect the significant societal and technological changes that have taken place in the last 20 years. Though predominantly focused on sport-specific objectives, the sport policy recognises, with varying degrees of specificity, that sport can support several development goals, including economic growth, national unity, or health promotion. Overall, the absence of national direction has left sport policymaking in the hands of the country's two major sport organisations, the Botswana National Sports Commission (BNSC) and the Botswana National Olympic Committee (BNOC). Though, in theory, the BNSC should be responsible for grassroots sport and the BNOC for elite sport, in practice the two organisations have overlapping mandates and programmes, and their activities both target elements of elite sport, sport for all and sport development [6,15-17]. Though two organisations operating in similar fields is not inherently problematic, this overlap combined with the existing rivalry between the two organisations severely restricts the sport sector's ability to work towards cross-cutting national objectives. Even for sport-specific goals, the lack of coherence is striking. For instance, in their most recent strategic documents, the BNOC and BNSC have even set differing medal targets for the Olympic Games [18].

Now that we have mapped the national policy landscape, we could engage in the next steps of Nilsson et al.'s [11] analytical framework, namely a screening analysis of policy objectives related to sport and in-depth analysis of policy interactions. However, given the disjointed nature of policy related to sport, we argue that it is difficult to engage in these steps in a meaningful way. Adequately screening the different objectives is made problematic by the nebulous definition of many of those objectives, and is further complicated by the unclear distinction between many objectives and instruments. Likewise, it is nearly impossible to analyse the interactions between the different policies. Even when objectives and/or instruments are reasonably well defined, there is little or no activity around the implementation of those instruments. For instance, there is "no documentation of implementation and evaluation" of physical activity promotion activities [19], and there appear to be no national actions taken to commercialise Botswana sport.

In short, the connections to sport in Botswana policy are too ill-defined to analyse the interactions between any given set of policy. Our goals moving forward are to begin addressing these issues. In the following, we wish to put forth specific policy objectives and instruments related to sport and sustainable development in Botswana. We further apply Nilsson's framework in a novel way, using it as a basis to outline a more coherent national sport for sustainable development policy. In that sense, this work should be understood as concerned primarily with horizontal policy coherence.

\section{Methodology}

\subsection{Design}

Stakeholder engagement and evidence building are critical for policy coherence [20]. In line with this, we rely on a mix of qualitative data to support our findings. Semi-structured interviews with individuals engaged in both Botswana sport 


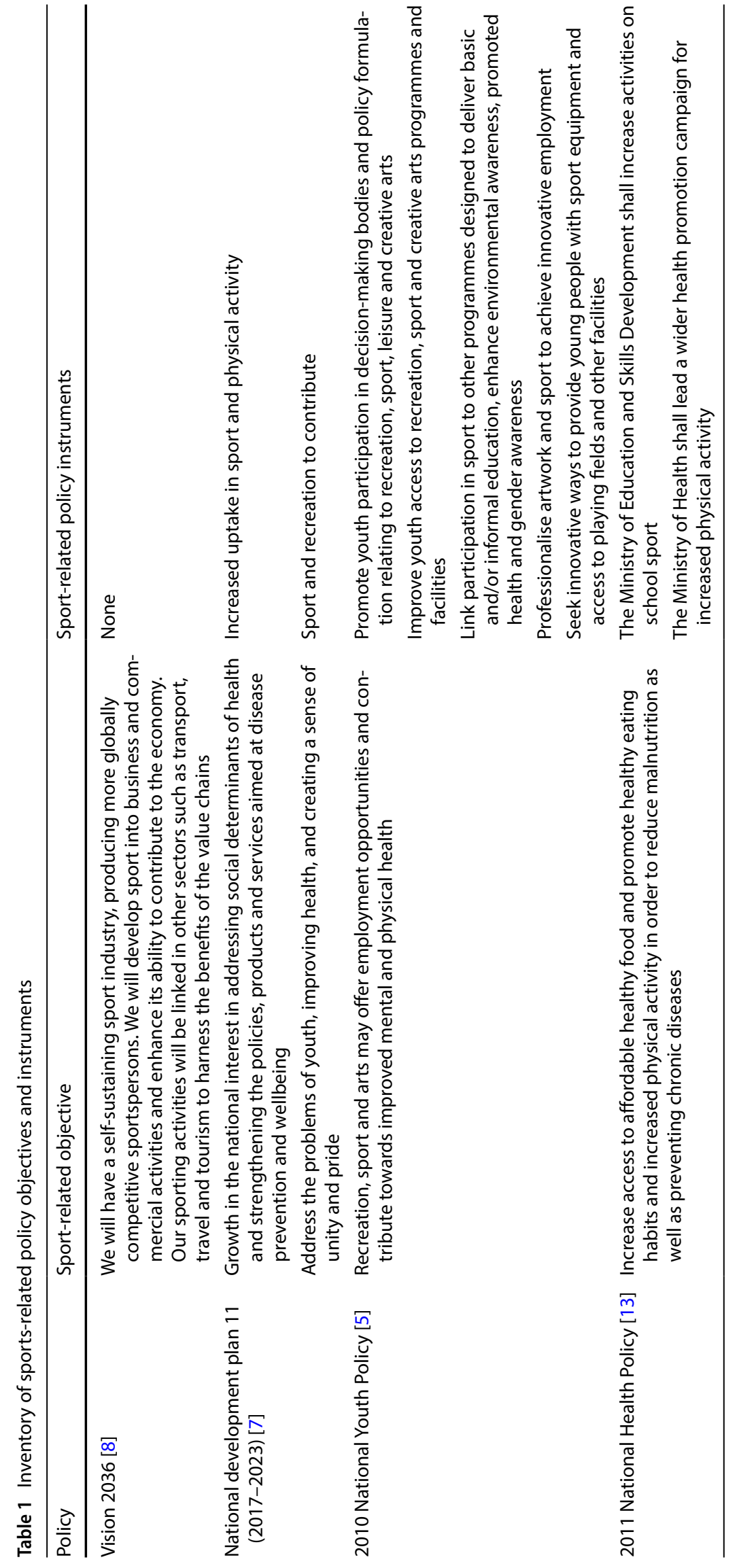


and other relevant fields (e.g. education, government, civil society, business) allowed us to identify policy objectives and instruments related to sport and sustainable development in Botswana. Nine interviews were conducted specifically for this paper. To support data saturation and validity, these main interviews are supported by results from previous interviews [including 21,22], as well as sources such as academic literature, newspaper articles or policy documents. Earlier interviews are included as previous research questions support the aims and scope of this paper. In other words, topics related to sport or sustainable development organically emerged in earlier work and support the findings presented here. The interviewee IDs and timelines for all main interviewees are shown in Table 2.

Purposive and snowball sampling approaches were used to source interviewees. Interviewees were approached by email or Facebook and generated through the first author's professional contacts in Botswana. Additional responses were generated via referrals from these contacts.

Ten Individuals were initially contacted, with six agreeing to participate in an interview. Another three individuals were sourced through referrals. In total, there were five men and four women. Interviewees were selected due to their connections to sport (e.g. as volunteers, administrators, athletes) as well as their engagement with other societal areas such as business, government, civil society or education. Involvement in both sporting and social spheres was considered essential. This allowed interviewees to have an understanding of the Botswana sporting environment and experience in other relevant fields, hence putting them in the position to be able to make informed connections between sport and sustainable development.

\subsection{Data collection}

The first author conducted semi-structured, online, face-to-face interviews in August 2020. In total, nine individuals were interviewed once, with interviews lasting between 37 and 67 min each. Before each interview, participants were explained the general purpose of the research and the researcher's interest in the topic. All participants were told that their statements would remain anonymous. The latter was done to allow interviewees to feel comfortable expressing opinions without fear of repercussion. Interviews took place over online meeting software (Zoom or WebEx) and, subject to interviewee approval, were digitally recorded. Notes were taken during and after the interviews. Following the literature on this method, interview guidelines were organised around a limited set of predetermined open-ended questions and probes emerged from the dialogue between interviewer and interviewee [23-26]. The interviews aimed to define where sport can contribute to Botswana's sustainable development objectives and map out policy instruments to support that contribution.

\subsection{Data analysis}

Interviews were transcribed verbatim into word processing software. The transcriptions were then analysed using MaxQDA 2020. MaxQDA was used to code the text and track the total amount of codes and connections between codes. Coding was done by the first author and cross-checked by the second author. Additionally, a first draft of the article was sent to all interviewees. These steps were taken to ensure the reliability and consistency of the overall results.

Codes emerged both deductively and inductively. Deductive codes were based on the sub-pillars of Vision 2036 and serve to identify the potential objectives related to sport and sustainable development. Codes related to policy instruments emerged inductively based on a process of thematic analysis. More precisely, the six-step approach of thematic

Table 2 Overview of main interviewees

\begin{tabular}{lll}
\hline ID & Gender & Occupational area \\
\hline M01 & Male & Politics/Government \\
M02 & Female & Sports \\
M03 & Male & Business \\
M04 & Male & Regional Development \\
M05 & Female & Sports \\
M06 & Female & Business \\
M07 & Male & Research/Academia \\
M08 & Female & Civil Society \\
M09 & Male & Politics/Government \\
\hline
\end{tabular}


analysis described by Braun and Clarke [27] was used to code and organise the data. This included becoming familiar with the descriptions, generating initial codes, searching for themes, reviewing potential themes, defining final themes, and writing the data. Coding trees for policy objectives and policy instruments are provided as Additional Files 1, 2, respectively.

\section{Defining objectives and instruments}

\subsection{Sporting events and economic development}

The economy is heavily dependent on the mining sector, which accounts for about one-third of Botswana's Gross Domestic Product (GDP) [28]. As a result, there is broad recognition that the country needs to diversify its sources of economic growth to ensure sustainable, long-term prosperity. Sport, both within the analysed policy documents and public discourse, is positioned as a solution to this challenge. For instance, Member of Parliament Ditlhapelo Keorapetse noted that sports contribute significantly to economies across the world and that it could do the same in Botswana [29]. The BNSC Vision 2028 strategic document even explicitly aims for sport to contribute $0.5 \%$ of the country's GDP [30]. This number is realistic, as studies from Europe show that sport can account for anywhere between $1-2 \%$ of Gross Value Added, as well as $1-2 \%$ of overall employment [31, 32].

Though the objective here is reasonably clear, the instruments to achieve it require additional definition. Many interviewees recognised that sport can foster important knock-on effects for related businesses or services. The presence of sporting events at a local facility can provide an opportunity for informal vendors or companies to offer products or services around the event, such as food, beverages, accommodation or merchandise. Indeed, events are a fundamental starting point for economic development in sport. Many commercial and employment activities start first by hosting an event:

People travel to go watch their teams. And when they do that, they are buying fuel to drive; they are buying beverages, they are going to book into some hotels, are going to buy apparel (M09).

Successful events can also contribute to generating employment, be it in terms of retail services, clerical work, or more modern occupations such as social media marketing. Yet, inherent to these knock-on effects is the assumption that reasonably well-attended events are taking place.

Hosting regular regional or international events can have substantial potential economic reverberations. These events bring in external visitors who help generate economic activity, promote Botswana as a touristic destination, and create opportunities for individuals to be employed in the delivery of events, both directly or indirectly. The focus should not be on hosting major, large-scale events, but rather on exploiting the opportunities presented by recurring or smallerscale events [33-38]:

I'm not talking about hosting these big major games that are held for two weeks, and then nothing happens. I'm talking about more long-term initiatives that are able to carry on throughout the year (...) Internationally some of these sports they actually have constant competition going on for athletes. In athletics, you have your marathons, you have your track events that happen on the global calendar throughout the year in terms of series that are being held, and that don't require a lot of money for nations to host, but you get a lot of participants coming into the country (M05).

This is not to imply that the country has not hosted regional or international sporting events. In the last few years, Botswana has welcomed the African Youth Games, the World Netball Youth Cup, and the International Working Group on Women in Sport. Further, the BNSC's Vision 2028 explicitly recognises event hosting as a priority for the sport sector. Rather, hosting events occurs on a happenstance, uncoordinated basis. There is no continuity between events and no overarching event hosting strategy for the country. A Sport Event Hosting Strategy (SEHS) would substantially contribute to managing and maximising Botswana's hosting potential. A SEHS can be roughly defined as a framework that regulates and coordinates the activities of various stakeholders to support the successful bidding and hosting for sport events [39]. As such, a SEHS helps identify the overall strategic goals of event hosting, the target events, the relevant stakeholders, tools and management processes. Numerous countries, including Canada, Denmark and Qatar, have developed such strategies against which Botswana could benchmark itself.

Better use of existing financial resources and infrastructure is needed to underpin any SEHS. For one, targeted investments in community recreational and sporting facilities are required. Though the government often invests in large, 
football-focused multi-purpose stadia [40], these buildings often turn into white elephants that are too expensive for organisations, clubs or communities to use regularly. Smaller facilities, and subsequent community ownership, are essential to give Botswana the ability to host local, regional and international sporting events across a wide range of sports. Findings from the tourism sector, as well as research on physical activity and sport in Botswana, support these recommendations [19, 41-43]. Current facilities should be exploited further and be more easily accessible to sport organisations:

There is too much resource guarding and not enough resource leveraging (M03).

Access to facilities is often fraught with obstacles that make it difficult for communities, organisations, or sports clubs to exploit them for their activities, never mind bid to host events. A prominent example of this comes from the African Youth Games. Significant investments were made to refurbish the University of Botswana pool, including integrating a heating and timing system. But, there was no long-term agreement made that would allow sport organisations to access the facility, inhibiting their ability to train or host competitions at that venue.

\subsection{Profesionalising sport}

Parallel to conversations about increasing the economic impact of sport, there has long been a discourse around the need to commercialise or professionalise sport in Botswana. In this context, this should be understood as moving the sport sector away from being predominantly volunteer-run and publicly-funded towards a self-sustaining sector with professional staff. Presently, the sector is almost entirely volunteer-run, and public money represents $80-90 \%$ of funding for the majority of sport organisations $[18,44]$. There are very few policy instruments that support sport organisations to generate sustainable, external funding and create additional employment in the sector. A law banning sponsorships from alcohol companies has severely impacted many sport organisations [45], and there is no legal framework to support the various commercial aspects of sport.

Economic topics such as merchandising, facility usage, employment or player agents are either not covered by legislation or are poorly enforced:

Our legislation and policies have not been reviewed or amended or upgraded to look into the value of sport could bring the economy (M09).

In turn, this dramatically limits the ability of sport organisations to generate sustainable external revenues. For instance, there is little enforcement of intellectual property rules or merchandise sales. Third-party vendors can print and sell merchandise for a particular club, and there is minimal regulation or enforcement against such ambush marketing. Similarly, there are limited frameworks for sport organisations to claim revenues from the sale of concessions (i.e. food and beverages) during their events. Proper legislation and enforcement are needed to protect the intellectual property of sport clubs, and public stadiums should formalise agreements with sport organisations regarding event revenue sharing.

Adjacent to this, there is a concern that sport administrators in the country do not have the necessary skills or training to exploit the opportunities presented by a fuller legal framework or a SEHS. Sport administrators are not sufficiently professionalised to offer a clear return on investment to sponsors or tap into all possible event hosting opportunities. As one interviewee put it,

I do not know whether we have got the relevant expertise to be able to run the different sports that we have here (M01).

Reflecting the volunteer-led nature of Botswana sport, many sport administrators have little or no training concerning sport management, or management more generally. There have been some positive developments in this area. The BNOC is active in the regular delivery of Sport Administrator Courses, and the University of Botswana has recently launched a Sport Management Programme. Nonetheless, there remains a concern that too few resources have been dedicated to training the individuals who manage sport in Botswana, and that the content of current courses does not reflect local realities. Required training for sport administrators can help address these concerns, and this training should exploit increasing local scholarship concerning the economic and social aspects of sport.

\subsection{Education and skill development}

There is increasing concern regarding educational outcomes in Botswana. Recently, there have been decreases in academic performance at various school levels and declining rates of pupils transitioning from junior to senior secondary 
education $[46,47]$. Furthermore, many graduates do not necessarily possess the soft skills (e.g. communication, teamwork, discipline) that are increasingly required in Botswana's modernising job market [48]. In light of this, there is greater recognition of the potential of sport to support education and skill development. To that end, many noted sport's ability to act as an attractive hook that could lead individuals into programmes where, around the sport sessions, educational offerings are provided. For instance, two interviewees spoke of how their sporting contexts have been used to provide employability-related training (e.g. CV writing, interviewing) with themselves or other athletes. Participation in sport itself can also support the development of several soft skills that are required for successful engagement in the job market. The interviewees mentioned a range of skills-including communication, leadership, confidence, discipline, autonomy and teamwork - that could be directly developed through sport participation. Yet the development of these skills is not automatic:

The coach has to deliberately expose players to them making decisions, developing a sense of autonomy, for example, deciding that the athlete is going to make this decision as opposed to the coach making the decision for the athlete (M07).

This intentionality and role of the sport educator are viewed as fundamental within the literature on sport and educational development $[49,50]$. However, in Botswana, the training of sport educators (physical education teachers and coaches) is lacking [51]. School sport organisations receive less than $3 \%$ of the annual sport budget [18], and physical education is not a mandatory subject in Botswana [19]. As a result, unspecialised and underequipped school teachers are often put in the position of delivering sport sessions:

We are leaving it to regular teachers, let's say a math teacher, we randomly say you can coach softball but are they really going to be coaching softball? They don't know how to (M05).

Similarly, many coaches are untrained volunteers. And, even when they do receive qualifications, there is often little focus on non-sport topics. This lack of sport-specific and non-sport pedagogical training impedes the ability of sport educators to foster soft skill development in their sessions. Yet these soft skills are increasingly required by a modernising Botswana job market and are essential to ensure sustainable economic growth in the twenty-first century.

To remedy this, standardised, widely implemented curriculums for sport educators are required, and these should include not only pedagogical approaches sport-didactical aspects but for soft skill development. There is a wealth of materials that could guide such a curriculum, including the LifeMatters programme, which has already been tested in the Botswana context [52]. The improvement of sport session delivery, especially at the school level, could have many beneficial side-effects that can help Botswana tackle some of its more recent formal education challenges. Quality school sport offers can make school itself more attractive, thereby increasing attendance [53]. Furthermore, sport sessions that integrate movement with academic or soft skill development can help boost academic achievement [54]

\subsection{Health promotion}

The physical and mental health benefits of sport and physical activity are well documented and proven. Amongst others, sport and physical activity can improve fitness, reduce the risk of certain diseases, help control weight, and can even support programmes that combat communicable diseases [55-57]. Beyond HIV/AIDS, which continues to be a challenge, Botswana has faced increasing rates of obesity and non-communicable diseases. Non-communicable diseases now account for about $30 \%$ of deaths in the country [58]. Promoting sport and physical activity participation is a direct way to counteract these trends:

If you cannot bring sport, your health bill is going to be high. More people will get sick, and it is a cost for the government as it pays for medication, hospitals and so on. If you put in sport in this picture, it automatically reduces the bill (M04).

Increasing sport and physical activity participation, first and foremost, requires consistent messaging and awarenessraising. In Botswana, sport is still perceived as a mostly recreational pursuit or hobby, and as a distraction from other more important activities such as schooling or work. For many Batswana, the connections between sport and the areas mentioned above, such as health or skill development, are not clear. This perception is rooted deep within the Setswana language, as the words for sport and play are used interchangeably, leading many to view sport not only as a pastime but as a waste of time. In the past, there has been a lack of public awareness programmes to communicate the benefits of sports [43]. To raise this awareness and challenge entrenched attitudes, broad public messaging is required. This 
messaging should focus on the benefits of sport participation as well as the inherent risks of non-participation. Highlighting health and financial risks such as obesity or health care costs can act as a risk discourse, or as as a moral technology through which "practices seeking to shape and guide people's conduct are deployed" [59]. Many interviewees further noted that websites or social media are underexploited in the sports sector, and these could act as wide-reaching, costeffective dissemination vehicles for awareness activities.

Though top-down awareness-raising is required to change entrenched attitudes, in parallel, there is a need for community investment and engagement in sport and physical activity. As discussed above, communities and local organisations often face significant barriers in accessing local facilities. A community development approach is needed to improve communication and collaboration among community partners involved in the provision of sport at the local level:

When you do a development, you want it to be owned by the community. This should not just be an entity for that is owned by the sports commission, or by the Ministry of Sports and Culture. You want it to be such that local community adopts it and can use it (M04).

Along with increased community involvement and ownership, there needs to be increased investment in community facilities. Again, smaller-scale indoor facilities or free, openly accessible outdoor sporting areas are paramount. There is a lack of well-maintained, open, safe recreational spaces. Recreational spaces often take the form of bare, empty plots of land, and these could be repurposed for community benefit:

These could be spaces in neighbourhoods used for sport activities if there was basic sporting infrastructure on them (M04).

For instance, these spaces could be used to install playgrounds, outdoor fitness equipment or netball courts. At present, as Botswana's most recent Physical Activity Report Card notes, the "lack of community sport facilities and equipment hinder participation in physical activity [19]. Competent, trained sport educators must complement these facilities and support the activities taking place there. Therefore, the issue of sport educator training is salient here again. Several studies identify coaches as one of the most relevant influences for continued commitment and motivation towards a sport or physical activity. The improved sport educator training described above also serves the purpose of increasing sport session quality and keeping more people engaged in physical activity.

\section{Connecting objectives and instruments}

In the above, we have analysed the coherence of current policy related to sport in Botswana and proposed a set of policy objectives and related instruments. However, policy coherence is first and foremost about avoiding redundancy and promoting synergies. Therefore, it is crucial to view our proposed objectives and instruments as a whole, which we do below in Table 3.

In this conceptualisation of a 'sport for sustainable development' policy, many of the redundancies or confusions of the existing policy landscape are removed. The overarching objectives are narrower, and underdeveloped references to objectives such as gender awareness or environmental protection are removed. Concrete instruments are proposed, and many of these instruments support multiple overarching objectives, creating a more coherent, synergistic policy. This reflects the reality that the different objectives and instruments do not exist in silos but are strongly interconnected. For instance, investing in small-scale, accessible recreational facilities would not only boost Botswana's ability to host sporting events but provide the needed infrastructure for increased physical activity participation.

Similarly, better and more widespread training of sport educators would support sport participation while allowing the development of crucial soft skills to be integrated within sport sessions. These overlaps reflect the notion of synergy that is inherent to the concept of policy coherence. Ultimately, identifying these overlaps allows policymakers to funnel limited resources towards instruments that can deliver benefits across a range of overarching objectives.

We do not contend that this is a full, comprehensive policy. Many sustainable development objectives that are connected with sport elsewhere, such as social inclusion or urban development, are not included here. These objectives did not regularly emerge in this study, though it is certainly worthwhile to explore the potential contributions of sport towards other areas in Botswana. We also cannot propose specific, measurable sub-objectives. Far more detailed work, research and consultation are required for that task. The proposed instruments are far more developed than in current policy and integrate many of the solutions that emerged through our research. Nonetheless, these require additional specification to address local needs and realities. Finally, our paper focuses on horizontal integration. Any national policy 
Table 3 Summary of propose policy objectives and related instruments

\begin{tabular}{|c|c|}
\hline Objective & Instruments \\
\hline \multirow[t]{4}{*}{ Support economic development and employment } & Develop an event hosting strategy for small-scale/regional sporting events \\
\hline & Invest in small-scale indoor facilities / outdoor public recreational facilities \\
\hline & $\begin{array}{l}\text { Implement and enforce a legal framework to underpin the commercial aspects of sport } \\
\text { (e.g. intellectual property, employment, etc.) }\end{array}$ \\
\hline & $\begin{array}{l}\text { Develop and implement sport administrator training that addresses local needs and } \\
\text { realities }\end{array}$ \\
\hline \multirow[t]{3}{*}{ Promote health and wellbeing } & Invest in small-scale indoor facilities / outdoor public recreational facilities \\
\hline & $\begin{array}{l}\text { Develop and implement a public awareness campaign to sensitise individuals about } \\
\text { the risks of NCDs and the benefits of sport/physical activity participation }\end{array}$ \\
\hline & $\begin{array}{l}\text { Standardise and implement training for sport educators, including as it relates to the } \\
\text { development of soft skills and other social issues }\end{array}$ \\
\hline Promote formal and informal (soft skill) education & $\begin{array}{l}\text { Standardise and implement training for sport educators, including as it relates to the } \\
\text { development of soft skills and other social issues }\end{array}$ \\
\hline
\end{tabular}

will need to consider how it (vertically) interacts with policies at the local, regional and international levels. Despite these constraints, our work illustrates two key elements. First, policy in Botswana related to sport and sustainable development is nebulous and lacks coherence, which in turn limits sport's contribution to Botswana's sustainable development. Second, engaging with stakeholders and current evidence empirically has allowed us to outline a more coherent, focused policy. We do not expect our 'sport for sustainable development' policy to be adopted whole cloth. However, we believe that our work can provide the impetus for the development of sport and sustainable development policy in Botswana and elsewhere.

\section{Conclusion}

Our study aimed to analyse the coherence of current policy related to sport in Botswana and propose policy objectives and instruments pertaining to sport and sustainable development. Using Nilsson's [11] analytical approach, we aimed to analyse the coherence of current policies as well as to outline a potentially more coherent 'sport for sustainable development' policy. To accomplish this, we relied on a mix of qualitative data, including interviews and literature. This allowed us to engage directly with local stakeholders while simultaneously underpinning our findings with relevant empirical evidence. In that sense, our methodology echoes the OECD guidelines on policy coherence, which suggest that both evidence and stakeholder engagement are critical [20].

From this, we find that sport should focus a narrower set of overarching sustainable development objectives, namely economic development, education, and health promotion. Numerous concrete policy instruments are likewise suggested, including investing in small-scale sport infrastructure, public awareness campaigns and better sport educator training. These instruments enhance the coherence of the overall policy as they help address multiple overarching objectives simultaneously.

Our work has significant policy implications. First and foremost, this work identifies redundancies within Botswana's policy landscape as it relates to sport and sustainable development. The results here can be of value to policymakers in the country looking to develop sport and address many of the overarching objectives identified in Vision 2036. Our use of Nilsson et al.'s framework [11] to support the design of coherent policy is also potentially useful. The way we have used this approach could be developed and replicated elsewhere, potentially providing policymakers and researchers with a simple yet rigorous tool for the development of coherent policy. For those working to implement or study sport as a tool for sustainable development, our approach provides a template for the development of a more cohesive policy. Nilsson et al.'s [11] framework has been principally used in environmental governance. Yet, it is clear that it applies to other sectors and, as we have shown here, can be used as a tool for both policy analysis and policy development. As sport continues to gain prominence as a tool for sustainable development, tools to develop and assess the coherence of a growing policy landscape are sorely required. 
Authors' contributions LM: the idea for the article, data collection, data analysis, writing. AAl: data collection, data analysis, critical revision. All authors read and approved the final manuscript.

Funding Open access funding provided by Projekt DEAL.

Availability of data and materials The datasets (e.g. interview transcriptions) generated during the current study are available from the corresponding author on reasonable request.

Code availability Not applicable.

Competing interests There are no conflicts to report.

Open Access This article is licensed under a Creative Commons Attribution 4.0 International License, which permits use, sharing, adaptation, distribution and reproduction in any medium or format, as long as you give appropriate credit to the original author(s) and the source, provide a link to the Creative Commons licence, and indicate if changes were made. The images or other third party material in this article are included in the article's Creative Commons licence, unless indicated otherwise in a credit line to the material. If material is not included in the article's Creative Commons licence and your intended use is not permitted by statutory regulation or exceeds the permitted use, you will need to obtain permission directly from the copyright holder. To view a copy of this licence, visit http://creativecommons.org/licenses/by/4.0/.

\section{References}

1. Lindsey I, Chapman T. Enhancing the Contribution of Sport to the Sustainable Development Goals. London: Commonwealth Secretariat; 2017.

2. Burnett C. Framing a 21 st century case for the social value of sport in South Africa. Sport Soc. 2019. https://doi.org/10.1080/17430 437.2019.1672153.

3. Lindsey I, Darby P. Sport and the Sustainable Development Goals: Where is the policy coherence? Int Rev Sociol Sport. 2019;54:793-812. https://doi.org/10.1177/1012690217752651.

4. Sherry E, Agius C, Topple C, Clark S. Measuring Alignment and Intentionality of Sport Policy on the Sustainable Development Goals. Melbourne; 2019.

5. Ministry of Youth, Sport and Culture. Revised National Youth Policy 2010. Gaborone; 2010.

6. Ministry of Youth. Sport and Culture. Gaborone: National Sport and Recreation Policy for Botswana; 2001.

7. Ministry of Finance and Development Planning. National development plan 11: April 2017-March 2023. [Gaborone]: Ministry of Finance and Development Planning; 2017.

8. Vision 2036 Presidential Task Team. Vision 2036: Achieving prosperity for all. Gaborone, Botswana: Lentswe La Lesedi (Pty) Ltd; 2016.

9. Sianes A. Shedding light on policy coherence for development: a conceptual framework. J Int Dev. 2017;29:134-46. https://doi. org/10.1002/jid.2977.

10. Nilsson M, Zamparutti T, Petersen JE, Nykvist B, Rudberg P, McGuinn J. Understanding policy coherence: analytical framework and examples of sector-environment policy interactions in the EU. Env Pol Gov. 2012;22:395-423. https://doi.org/10.1002/eet.1589.

11. Nilsson M, Wiklund H, Finnveden G, Jonsson DK, Lundberg K, Tyskeng S, Wallgren O. Analytical framework and tool kit for SEA follow-up. Environ Impact Assess Rev. 2009;29:186-99. https://doi.org/10.1016/j.eiar.2008.09.002.

12. Linder SH, Peters BG. Instruments of Government: Perceptions and Contexts. J Pub Policy. 1989;9:35-58.

13. Republic of Botswana. National Health Policy Towards a Healthier Botswana. Gaborone; 2011.

14. BOPA. National sport, recreation policy sufficien. 2014. http://www.dailynews.gov.bw/mobile/news-details.php?nid=9102\&flag=5. Accessed 21 Aug 2020.

15. Botswana National Olympic Committee. Botswana Long Term Athlete Development 2020 and Beyond Strategic Plan. Gaborone; 2015.

16. Botswana National Olympic Committee. About The BNOC. 2014. http://botswananoc.org/about-bnoc. Accessed 5 Sep 2016.

17. Botswana National Sports Commission. About the BNSC. 2016. http://www.bnsc.co.bw/bnsc-about-us.php?pagename=About\%20BNS C. Accessed 2 May 2019.

18. Moustakas L, Tshube T. Sport Policy in Botswana. Int J Sport Policy Politics. 2020. https://doi.org/10.1080/19406940.2020.1832556.

19. Tladi DM, Monnaatsie M, Shaibu S, Sinombe G, Mokone GG, Gabaitiri L, et al. Results from Botswana's 2018 Report Card on Physical Activity for Children and Youth. J Phys Act Health. 2018;15:S320-2. https://doi.org/10.1123/jpah.2018-0420.

20. Better Policies for Sustainable Development 2016: OECD; 2016.

21. Moustakas L. The Influence of Local NGOs on Botswana's Human Development in the 21st Century: a Case Study: Unpublished; 2019.

22. Moustakas L. Big games in small places: the african youth games and organizational capacity in botswana sport federations. Eur Sport Manag Q. 2020. https://doi.org/10.1080/16184742.2020.1849341.

23. Barriball KL, While A. Collecting data using a semi-structured interview: a discussion paper. J Adv Nurs. 1994;19:328-35.

24. Turner DW III. Qualitative interview design: a practical guide for novice investigators. Qualitat Rep. 2010;15:754-60.

25. Dicicco-Bloom B, Crabtree BF. The qualitative research interview. Med Educ. 2006;40:314-21.

26. Bryman A. Social research methods. 4th ed. Oxford: Oxford Univ. Press; 2012.

27. Braun V, Clarke V. Thematic analysis. In: Cooper H, Camic PM, Long DL, Panter AT, Rindskopf D, Sher KJ, editors. APA handbook of research methods in psychology, Vol 2: Research designs: Quantitative, qualitative, neuropsychological, and biological. Washington: American Psychological Association; 2012. p. 57-71. doi:https://doi.org/10.1037/13620-004. 
28. Jefferis K. The role of TNCs in the extractive industry of Botswana. Transnational Corporations. 2009;18.

29. Kgangkenna D. Commercialise sport-Keorapetse. Sunday Standard. 17.12.2019.

30. Botswana National Sports Council. Strategy Vision 2028. Gaborone; 2013.

31. SportsEconAustria. Study on the Contribution of Sport to Economic Growth and Employment in the EU. Brussels; 2012.

32. Eurostat. Employment in Sport. Brussels; 2019.

33. Walo M, Bull A, Breen H. Achieving economic benefit at local events: a case study of a local sports event. J Festival Manage Event Tourism. 1996;4:95-106.

34. Agha N, Taks M. A theoretical comparison of the economic impact of large and small events. Int J Sport Fin. 2015;10:103-21.

35. Taks M, Kesenne S, Chalip L, Green BC, Martyn S. Economic Impact Analysis Versus Cost Benefit Analysis: The Case of a Medium-Sized Sport Event. Int J Sport Finance. 2011;6:187-203.

36. Wilson R. The economic impact of local sport events: significant, limited or otherwise? A case study of four swimming events. Managing Leisure. 2006;11:57-70. https://doi.org/10.1080/13606710500445718.

37. Daniels MJ, Norman WC. Estimating the economic impacts of seven regular sport tourism events. J Sport Tourism. 2003;8:214-22. https ://doi.org/10.1080/1477508032000161528.

38. Veltri FR, Miller JJ, Harris A. Club sport national tournament: economic impact of a small event on a mid-size community. Recreat Sports J. 2009;33:119-28. https://doi.org/10.1123/rsj.33.2.119.

39. Chappelet J-L, Lee KH. The Emerging Concept of Sport-Event-Hosting Strategy: Definition and Comparison. J Global Sport Manage. 2016;1:34-48. https://doi.org/10.1080/24704067.2016.1177354.

40. Kgautlhe C. Botswana: Construction of Tsabong Stadium to Start Soon. 2019. https://allafrica.com/stories/201903120167.html. Accessed 21 Aug 2020.

41. Nare AT, Musikavanhu GM, Chiutsi S. Tourism diversification in Botswana - a stakeholder perspective. Afr J Hospital Tour Leisure. 2017;6:155-65.

42. Tselaesele N, Bulala T, Marumo D. Gender analysis study on participation of girls and women in sport and leadership in Botswana. Gaborone; 2017.

43. Amusa L, Toriola OM, Onyewadume LU, Dhaliwal HS. Perceived barriers to sport and recreation participation in Botswna. Afr J Phys Health Educat Recreat Dance. 2008;14:115-29.

44. Botswana ML. In: Hallmann K, Fairley S, editors. Sports Volunteers Around the Globe. Cham: Springer International Publishing; 2018. p. 33-42.

45. Kolantsho C. Alcohol ban costs Botswana COSAFA Cup. Mmegi. 24.06.2016.

46. Ministry of Finance and Development Planning. National Development Plan 11. Gaborone; 2016

47. Makwinja V. Rethinking education in botswana: a need to overhaul the botswana education system. J Int Educat Res. 2017;13:45-58.

48. Human Resource Development Council of Botswana. Priority Skills and Employment Trends. Gaborone; 2019.

49. Cope E, Bailey R, Parnell D, Nicholls A. Football, sport and the development of young people's life skills. Sport in Society. 2017;20:789-801. https://doi.org/10.1080/17430437.2016.1207771.

50. Bailey R. Physical education and sport in schools: a review of benefits and outcomes. J Sch Health. 2006;76:397-401. https://doi.org/10. 1111/j.1746-1561.2006.00132.x.

51. Tshube T, Hanrahan SJ. Coaching and coach education in Botswana. Int Sport Coach J. 2018;5:79-83. https://doi.org/10.1123/ iscj.2017-0088.

52. Hanrahan S, Tshube T. Training coaches in positive youth development in Botswana. J Sci Med Sport. 2017;20:e50-1. https://doi. org/10.1016/j.jsams.2017.01.137.

53. Bailey R. Evaluating the relationship between physical education, sport and social inclusion. Educat Rev. 2005;57:71-90. https://doi. org/10.1080/0013191042000274196.

54. Zach S, Shoval E, Lidor R. Physical education and academic achievement-literature review 1997-2015. J Curricul Stud. $2017 ; 49: 703-21$. https://doi.org/10.1080/00220272.2016.1234649.

55. World Health Organization. Physical activity. 2018. https://www.who.int/news-room/fact-sheets/detail/physical-activity. Accessed 14 Jul 2020.

56. Melzer K, Kayser B, Pichard C. Physical activity: the health benefits outweigh the risks. Curr Opin Clin Nutrit Metabol Care. 2004;7:641-7.

57. Parnell D, Pringle A. Football and health improvement: an emerging field. Soccer Soc. 2016;17:171-4. https://doi.org/10.1080/14660 970.2015 .1082753

58. Silva R, Menon V, Mosime W, Lang E. Noncommunicable diseases: the payoffs of investing early in prevention efforts: policy brief-Botswana. Washington; 2015.

59. McDermott L. A governmental analysis of children "At Risk" in a world of physical inactivity and obesity epidemics. Sociol Sport J. 2007;24:302-24. https://doi.org/10.1123/ssj.24.3.302.

Publisher's Note Springer Nature remains neutral with regard to jurisdictional claims in published maps and institutional affiliations. 